\title{
ON THE EXISTENCE OF EXCEPTIONAL FIELD EXTENSIONS
}

\section{BY ROBERT GILMER ${ }^{1}$ AND WILLIAM HEINZER}

Communicated by G. D. Mostow, January 15, 1968

Let $F$ be a field of characteristic $p \neq 0$ and let $K$ be an algebraic field extension of $F$. Let $K_{i}$ denote the subfield of $K$ of elements purely inseparable over $F, K_{8}$ the subfield of separable elements, and $K^{n}$ the normal closure of $K / F$. We say that $K / F$ splits if $K=K_{i} K_{s}$ and following Reid's terminology in [2], $K$ is called an exceptional extension of $F$ provided $K_{i}=F$ and $K_{s} \neq K$.

LEMma 1. $K / F$ splits if and only if $K_{i}=\left(K^{n}\right)_{i}$.

Proof. If $K / F$ splits it follows easily that $K_{i}=\left(K^{n}\right)_{i}$. Conversely assume that $K_{i}=\left(K^{n}\right)_{i}$. Then $K^{n} / K$ is separable normal and hence a Galois extension. Since a normal extension splits we have $K^{n}$ $=\left(K^{n}\right)_{i}\left(K^{n}\right)_{s}$ and if $a \in K, a=\sum a_{\alpha} e_{\alpha}$ with $a_{\alpha} \in\left(K^{n}\right)_{s}$ and $\left\{e_{\alpha}\right\}$ a linearly independent set of elements of $\left(K^{n}\right)_{i}=K_{i}$ over $F$. If $\sigma$ is an automorphism of $K^{n} / K$ then $\sigma(a)=a$ implies that $\sum\left(\sigma\left(a_{\alpha}\right)-a_{\alpha}\right) e_{\alpha}=0$. But $K_{i}$ and $\left(K^{n}\right)_{e}$ are linearly disjoint over $F$ so that $\left\{e_{\alpha}\right\}$ is linearly independent over $\left(K^{n}\right)_{s}$. Hence $\sigma\left(a_{\alpha}\right)=a_{\alpha}$ and we have $a_{\alpha} \in K$ $\cap\left(K^{n}\right)_{\mathrm{q}}=K_{\mathrm{s}}$. Thus $K=K_{\mathrm{s}} K_{\mathrm{i}}$.

THEOREM 2. If $K / F$ is a simple extension then $K / F$ splits if and only if $K^{n} / F$ is simple.

Proof. If $K / F$ splits then by Lemma $1, K_{i}=\left(K^{n}\right)_{i}$ and it is clear that $K^{n} / F$ is also simple.

If $K^{n} / F$ is simple then $K / F$ and $\left(K^{n}\right)_{i} / F$ are simple. Let $f(X)$ be the minimum polynomial of $t$ over $F$, where $t$ is chosen such that $K=F(t)$. Then $K^{n}$ is the splitting field of $f(X)$ and we have

(a) $\exp f(X)=\exp \left(K^{n}\right)_{i}$,

(b) $p^{\exp f(X)}=\left[K: K_{\mathrm{a}}\right]$.

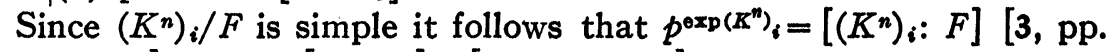
120-123]. Hence $\left[K: K_{s}\right]=\left[\left(K^{n}\right)_{i} K_{8}: K_{8}\right]$ and since $K \subseteq\left(K^{n}\right)_{i} K_{\mathrm{e}}$ we have $\left(K^{n}\right)_{i} K_{i}=K$ and $\left(K^{n}\right)_{i}=K_{i}$. By Lemma $1, K / F$ splits.

Our next lemma gives a method for constructing exceptional field extensions.

1 The first author received partial support from the National Science Foundation while this research was being done. 
LEMMA 3. Let $a, b$, and $s$ be elements of an algebraic extension field of $F$ with $a$ and $b$ purely inseparable over $F$, s separable over $F$ and not in $F$. Let $t=a+b s$ and $K=F(t)$. Then $F(a, b)=\left(K^{n}\right)_{i}$ and $F(a, b) / F$ is generated by the coefficients of the minimum polynomial for $t$ over $F(a, b)$.

PROOF. ${ }^{2}$ Let $s=s_{1}, s_{2}, \cdots, s_{n}$ be a complete set of conjugates of $s$ over $F$ and let $t_{i}=a+b s_{i}$. If $e$ is a nonnegative integer such that $a^{p^{0}}, \quad b^{p^{0}} \in F$, then $F\left(t_{i}^{p^{e}}\right)=F\left(s_{i}^{p^{0}}\right)=F\left(s_{i}\right)$. Hence $F\left(s_{1}, \cdots, s_{n}\right)$ $\subseteq F\left(t_{1}, \cdots, t_{n}\right)$. Also $b=\left(t_{1}-t_{2}\right)\left(s_{1}-s_{2}\right)^{-1}$ so that $b$, and hence $a$, are in $F\left(t_{1}, \cdots, t_{n}\right)$. It follows that $F\left(t_{1}, \cdots, t_{n}\right)=F(a, b) \otimes F\left(s_{1}, \cdots, s_{n}\right)$. And since the $t_{i}$ are con ugates over $F$, we have $F\left(t_{1}, \cdots, t_{n}\right)=K^{n}$ and $F(a, b)=\left(K^{n}\right)_{i}[1$, p. 50]. The minimum polynomial for $t$ over $F(a, b)$ is $g=\prod_{i=1}^{n}\left(X-t_{i}\right)$. If $F_{0}$ is the subfield of $F(a, b)$ obtained by adjoining the coefficients of $g$ to $F$, then $F_{0} / F$ is purely inseparable and $K^{n} / F_{0}$ is separable. Therefore, $F_{0}=\left(K^{n}\right)_{i}=F(a, b)$.

REMARK 4. Reid calls a separable field extension $E / F$ realizable if there exists an exceptional extension $K / F$ with $E=K_{8}$ [2]. Using Lemma 3 we can show that when $F / F^{p}$ is not simple then any proper separable extension of $F$ is realizable.

TheOREM 5. Let $K / F$ be normal and inseparable, but not purely inseparable. Then $K / F$ is simple if and only if every subextension of $K / F$ splits.

Proof. If $K / F$ is simple and $E$ is an intermediate field then we can take $E^{n} \subseteq K$. Hence $E^{n} / F$ is simple and by Theorem $2, E / F$ splits. Conversely if $K / F$ is not simple then $K_{i} / F$ is not simple. Hence there exist $a, b \in K_{i}$ such that $F(a, b) / F$ is not simple. We choose $s \in K_{s}-F$ and set $t=a+b s$. If $E=F(t)$ then by Lemma 3, $F(a, b) \subseteq E^{n}$ so that $E^{n} / F$ is not simple. Hence by Theorem $2, E / F$ does not split.

Our next result gives necessary and sufficient conditions that a given normal inseparable extension $K / F$ contain intermediate fields which are exceptional over $F$.

Theorem 6. Let $K / F$ be normal and inseparable but not purely inseparable. Let $E$ be the maximal purely inseparable subfield of $K / F$ of exponent one. Then $E / F$ is simple if and only if $K / F$ contains no exceptional subextensions.

Proof. If $K / F$ contains an exceptional subextension then $K$ contains an element $t$ such that $F(t) / F$ is exceptional of exponent one.

2 The proof of Lemma 3 indicated here is that of H. F. Kreimer; it simplifies an earlier proof due to the authors. 
Thus $F(t) / F$ does not split and $F(t)^{n}$ is not simple by Theorem 2 . Hence $\left(F(t)^{n}\right)_{i}$ is purely inseparable of exponent one and not simple. Thus $E / F$ is not simple.

To prove the converse we assume that $E / F$ is not simple and choose $a, b \in E$ such that $F(a, b) / F$ is not simple. Let $s \in K_{s}-F$ and, as in Lemma 3 , set $t=a+b s$. Then $F(t) / F$ does not split and $F(a, b)$ $=\left(F(t)^{n}\right)_{i}$. Moreover, $F\left(t^{p}\right)=F(s)$ is separable over $F$. Thus if $F(t) \cap F(a, b)$ properly contained $F$ then $F(t) / F$ would necessarily split. Hence $F(t)_{i}=F$ and $F(t) / F$ is exceptional.

Corollary 7. If $F(t) / F$ is inseparable but not purely inseparable and if $f=\sum_{i=0}^{r} a_{i} X^{i p^{\circ}}$ is the minimum polynomial for $t$ over $F$, where $e=\exp f$, then $F(t) / F$ is exceptional if and only if $F\left(\left\{a_{t}^{1 / p}\right\}_{0}^{r}\right) / F$ is not simple.

Proof. Sufficiency follows as in Theorem 2. Necessity follows from Theorem 6 and the fact that $F\left(\left\{a_{i}^{1 / p}\right\}_{0}^{r}\right)$ is the maximal purely inseparable subfield of exponent one of $F(t)^{n} / F$.

In view of Theorem 6 , if there exists a purely inseparable extension $L$ of $F$ such that $L / F$ is not simple and such that $E / F$ is simple where $E$ is the maximal subfield of $L / F$ of exponent one, then there exists a normal extension $K$ of $F$ such that $K / F$ is not simple, but there are no intermediate exceptional extensions. If we take $F=P(X, Y, Z)$ where $P$ is a perfect field and where $\{X, Y, Z\}$ is algebraically independent over $P$, and if $L=F\left(X^{1 / p}, X^{1 / p^{2}}+Y^{1 / p}, X^{1 / p^{2}} Z^{1 / p}\right)$, then it can be shown that $E=F\left(X^{1 / p}\right)$, providing the desired example.

\section{REFERENCES}

1. N. Jacobson, Lectures in abstract algebra. Vol. 3, Van Nostrand, Princeton, N. J., 1964.

2. J. D. Reid, A note on inseparability, Michigan Math. J. 13 (1966), 219-223.

3. B. L. van der Waerden, Modern algebra. Vol. 1, Unzar, New York, 1953.

Florida State University and

Louisiana State University, Baton Rouge 